\title{
Emergency Department Visits and Trends Related to Cocaine, Psychostimulants, and Opioids in the United States, 2008-2018
}

\author{
Leslie W. Suen ( $\sim$ leslie.suen@ucsf.edu ) \\ University of California, San Francisco \\ Thibaut Davy-Mendez \\ University of North Carolina at Chapel Hill \\ Kathy T. LeSaint \\ University of California, San Francisco \\ Elise D. Riley \\ University of California, San Francisco \\ Phillip Coffin \\ San Francisco Department of Public Health
}

\section{Research Article}

Keywords: amphetamines, cocaine, stimulants, emergency department, opioids, overdose

Posted Date: September 27th, 2021

DOI: https://doi.org/10.21203/rs.3.rs-821189/v1

License: (9) (1) This work is licensed under a Creative Commons Attribution 4.0 International License. Read Full License

Version of Record: A version of this preprint was published at BMC Emergency Medicine on February 4th, 2022. See the published version at https://doi.org/10.1186/s12873-022-00573-0. 


\section{Abstract \\ Background}

Drug-related emergency department (ED) visits are escalating, especially for stimulant use (i.e., cocaine and psychostimulants such as methamphetamine). We sought to characterize rates, presentation, and management of US ED visits related to cocaine and psychostimulant use, compared to opioid use.

\section{Methods}

We used 2008-2018 National Hospital Ambulatory Medical Care Survey data to identify a nationally representative sample of ED visits related to cocaine and psychostimulant use, with opioids as the comparator. We excluded visits related to $\geq 2$ of the three possible drug categories. We estimated annual rate trends using unadjusted Poisson regression; described demographics, presenting concerns, and management; and determined associations between drugtype and presenting concerns (categorized as psychiatric, neurologic, cardiopulmonary, and drug toxicity/withdrawal) using logistic regression, adjusting for age, sex, race/ethnicity, and homelessness.

\section{Results}

Cocaine-related ED visits did not significantly increase, while psychostimulant-related ED visits increased from 2008 to 2018 (2.2 visits per 10,000 population to 12.9 visits per 10,000 population; $p<0.001$ ). Cocaine-related ED visits had higher usage of cardiac testing, while psychostimulant-related ED visits had higher usage of chemical restraints than opioid-related ED visits. Cocaine- and psychostimulant-related ED visits had greater odds of presenting with cardiopulmonary concerns (cocaine adjusted odds ratio [aOR] 2.95, 95\% Cl 1.70-5.13; psychostimulant aOR 2.46, 95\% Cl 1.42-4.26), while psychostimulant-related visits had greater odds of presenting with psychiatric concerns (aOR 2.69; $95 \% \mathrm{Cl} 1.83-3.95)$ and lower odds of presenting with drug toxicity/withdrawal concerns (aOR $0.47,95 \% \mathrm{Cl} 0.30-0.73$ ) compared to opioid-related ED visits.

\section{Conclusion}

Presentations for stimulant-related ED visits differ from opioid-related ED visits: compared to opioids, ED presentations related to cocaine and psychostimulants are less often identified as related to drug toxicity/withdrawal and more often require interventions to address acute cardiopulmonary and psychiatric complications.

\section{Introduction}

Emergency department (ED) visits related to drug overdose are escalating, especially during the COVID-19 pandemic. ${ }^{1}$ While national attention has focused on opioids in the escalating overdose crisis, hospitals and EDs across the United States (US) are also facing an expanding burden of visits related to stimulant use. ${ }^{2-5}$ Stimulant-related ED visits are largely attributed to the use of controlled substances such as cocaine and psychostimulants (e.g., methamphetamine) ${ }^{6-9}$ Cocaine has been a major contributor to stimulant-related harms since at least $2011,{ }^{9}$ though in recent years, the US has seen a surge in methamphetamine use and related complications. ${ }^{10}$ In addition to stimulant-related ED visits increasing nationwide, ${ }^{5}$ stimulant-related overdose deaths have also rapidly increased since $2010 .{ }^{11}$ Overdose deaths from stimulants now outnumber overdose deaths attributed to prescription opioids and heroin. ${ }^{8}$

Prior ED studies have mostly focused on ED visits related to stimulant overdose. ${ }^{1,4,5}$ However, acute stimulant toxicity is not always recognized as an overdose due to symptom variability, ${ }^{12}$ and these studies do not address ED visits related to chronic complications of drug use. There is a need to broadly examine the characteristics, presentations, and clinical management of all stimulant-related ED visits, not only visits that are identified as related to overdose. Presentations from stimulant toxicity also show wide variability, affect multiple organ systems, and are not as readily attributed to substance use. ${ }^{4,13-16}$ Cocaine toxicity is strongly associated with cardiovascular complications, ${ }^{13,17}$ while psychostimulant toxicity is also associated with cardiac complications, as well as acute psychosis and agitation. 3,4,14 Little is known about which presenting concerns dominate for psychostimulant-related ED visits, and few studies have examined how ED presentations for cocaine and psychostimulant use vary compared to opioid use. A better understanding is needed, as how patients present to ED visits has significant implications on clinical management and ED resource utilization.

We sought to describe the annual trends and characteristics of cocaine- and psychostimulant-related ED visits, with opioids-related ED visits as the comparison group. We also evaluated whether chief presenting concerns during ED visits differ between these groups of drug-related ED visits.

\section{Methods}

\section{Study design and setting}

We conducted a secondary analysis using 2008-2018 data from the National Hospital Ambulatory Medical Care Survey (NHAMCS), a federally funded, nationally representative dataset of ED visits collected annually by the Centers for Disease Control and Prevention National Center for Health Statistics 
(NCHS). ${ }^{18}$ Each year, trained hospital and NCHS staff abstract visit data from medical records using standardized recording forms, including information on demographics, reasons for visit (RFV), vital signs, diagnostic testing, administered medications, diagnoses rendered, among others. NHAMCS uses a multi-stage probability design and applies survey weights to ED visits to obtain a nationally representative sample of ED visits to non-federal, general, short-stay acute care hospitals in the US. ${ }^{18,19}$

For each ED visit in NHAMCS in 2008-2013, up to three associated diagnosis codes, up to three RFV codes, and up to eight administered medications were available. ${ }^{20,21}$ For $2014-2018$, up to five associated diagnosis codes, up to five RFV codes, and up to thirty administered medications were available. ${ }^{22}$ For consistency, we used only the top three listed diagnosis codes, top three listed RFV codes as their chief presenting concerns, and top eight listed administered medications.

\section{Selection of Emergency Department Visits}

The sample included all ED visits made by adults (18 years or older) with the visit related to cocaine use, psychostimulant use, or opioid use, based on listed International Classification of Diseases Clinical Modification (ICD-CM) visit diagnosis codes. We included any visit that had any ICD-9-CM/ICD-10-CM code related to drug dependence, abuse, or poisoning for cocaine use, psychostimulant use, or opioid use in any of the top three listed diagnoses codes (see Appendix 1 for complete list of $I C D$ codes used). ${ }^{23,24}$ We excluded $I C D$ codes that included "in remission" to capture visits related to active drug use. We adapted this approach from the Centers for Medicare and Medicaid Services and other similar studies using national data. ${ }^{2,4,25}$ To make groups mutually exclusive and to allow for comparisons, we excluded any visits involving two or more of the three drug-related diagnosis groups (cocaine, psychostimulant, or opioid), which were approximately $5 \%$ of unweighted eligible visits. We also excluded visits identified by NHAMCS as follow up visits and visits where the patient was seen at the same hospital within the preceding 72 hours to limit counting repeat visits for the same illness episode.

\section{Measurements and Outcomes}

The primary exposure of interest was the type of drug associated with the ED visit, as defined above (cocaine, psychostimulant, or opioid). The primary outcomes of interest were the chief presenting concerns, measured as dichotomous variables if visit concerns were related to: (1) psychiatric; (2) neurologic; (3) cardiopulmonary; or (4) drug toxicity/withdrawal concerns. Visits could contribute to more than one category of presenting concerns. For chief presenting concerns, NHAMCS uses an RFV coding scheme developed by NCHS, where RFV is defined as "the patient's complaint(s), symptom(s), or other reasons for this visit." ${ }^{20}$ We adapted the RFV coding scheme to measure if any of the top three RFV codes associated with each visit were related to psychiatric, neurologic, cardiopulmonary, and drug toxicity/withdrawal categories of RFV codes. ${ }^{26}$ For example, psychiatric presenting concerns included RFV codes such as "depression" and "suicide attempt." Cardiopulmonary concerns included codes such as "chest pain," "shortness of breath," and "respiratory arrest." Drug toxicity/withdrawal concerns included "drug detoxification," "accidental poisoning," and "adverse effects of drug use" such as unintentional overdose (see Appendix 2 for RFV code categorization).

For each category of drug-related visits (cocaine, psychostimulant, or opioid), we described patient characteristics including age, sex, race/ethnicity, primary payer, homelessness, and multimorbidity (defined as the presence of two or more comorbidities assessed by NHAMCS). ${ }^{27}$ We used the imputed measure of race/ethnicity provided by NHAMCS, which adjusts for $16-18 \%$ missingness of race/ethnicity data extracted from the medical chart. ${ }^{21,22}$ We assessed hospital-level factors including urban location, US census region, and safety-net status, defined per NCHS criteria as having either $>30 \%$ of visits with the primary payer being Medicaid or uninsured, or having $>40 \%$ of visits from combined Medicaid and uninsured. ${ }^{29}$

To evaluate characteristics of clinical management, we assessed diagnostic testing, administered medications, and disposition. As above, we analyzed the top eight administered medications, using the drug classification scheme developed by NCHS. ${ }^{21,30}$ We reviewed drug codes available and included any drug codes that could be classified as "atypical antipsychotics," "benzodiazepines," "naloxone," or "opioids." We additionally examined whether visits were concurrently associated with alcohol-related and other drug-related diagnoses, including cannabis, sedatives/hypnotics, etc. (Appendix 1)

\section{Statistical analysis}

We applied survey sample weights to yield an unbiased national estimate of ED visit percentages and characteristics. ${ }^{19}$ We calculated annual ED visit rates by dividing the weighted number of visits each year by the US Census Bureau estimates of civilian, noninstitutionalized adults aged 18 and older. ${ }^{19}$ We conducted a Poisson regression analysis using visit year as the ordinal predictor to test for significant trends in visits over time. We described characteristics of cocaine-, psychostimulant-, and opioid-related visits using bivariate Pearson chi-squared tests. We used multivariable logistic regression to evaluate whether type of drug-related visit was associated with specific categories of chief presenting concerns, using separate models for each category. We adjusted for age, sex, race/ethnicity, and homelessness, as these factors could be associated with increased morbidity and introduce potential confounding. All reported estimates were robust, defined by NCHS as an unweighted count in each group of $\geq 30$ visits and/or a relative standard error of $\leq 30 \% .{ }^{18,31}$ We completed all analyses using svy routine commands within Stata/MP, version 16.0 (StataCorp LLC). ${ }^{19,32}$ All p-values were two-sided and a p-value $<0.05$ was used to determine statistical significance. The University of California, San Francisco Institutional Review Board exempted this study from review.

\section{Results}

Annual Rate Trends of Drug-Related Emergency Department Visits 
The study sample included 1,576,000 unweighted ED visits between 2008 to 2018, which was representative of 7,121,000 weighted ED visits. The rate of cocaine-related ED visits increased from 6.6 visits per 10,000 population $(95 \% \mathrm{Cl} 3.9-9.3)$ in 2008 to 8.9 visits per 10,000 population ( $95 \% \mathrm{Cl} 4.7-13.1)$ in 2018 , though this increase was not statistically significant ( $p=0.23$ for test of trend). Rates of psychostimulant-related ED visits increased from 2.2 visits per 10,000 population $(95 \% \mathrm{Cl} 0.8-3.7)$ to 12.9 visits per 10,000 population $(95 \% \mathrm{Cl} 7.3-18.4)(\mathrm{p}<0.001)$. The increase in ED visits was greatest for opioids, where rates of opioid-related ED visits increased from 6.0 visits per 10,000 population $(95 \% \mathrm{Cl} 3.7-8.2)$ in 2014 to 24.8 visits per 10,000 population ( $95 \% \mathrm{Cl}$ 18.0-31.5) in $2018(p<0.001)$ (Fig. 1).

\section{Characteristics of Drug-Related Emergency Department Visits}

In unadjusted analysis, psychostimulant- and opioid-related visits had comparable age and race/ethnicity distributions, whereas patients with cocainerelated visits were more likely to be among those $\geq 40$ years of age and identifying as Black $(p<0.001$ for both characteristics) (Table 1$)$. Patients with cocaine- and psychostimulant-related visits were more likely to be experiencing homelessness compared to patients with opioid-related visits (cocaine $10 \%$, psychostimulant $12 \%$, opioid $4 \% ; p<0.001)$. Cocaine-related visits were predominantly in the South $(49 \%)$, while psychostimulant-related visits were more concentrated in the West (60\%), and opioid-related visits were spread out throughout all regions. Cocaine and psychostimulant-related visits were slightly more concentrated at hospitals in urban areas than opioid-related visits (cocaine $96 \%$, psychostimulant 91\%, opioid 88\%; $p=0.03$ ). 
Table 1

Demographic characteristics of national emergency department visits related to cocaine, psychostimulant, opioid use, 2008-2018

Weighted $\%(95 \% \mathrm{Cl})$

Cocaine-related visits

$(n=1,406,000)$

\section{Psychostimulant-related visits}

$(n=1,590,000)$
Opioid-related visits

$(n=4,125,000)$

\section{Age, years}

\begin{tabular}{llll}
$18-29$ & $12(9,17)$ & $38(31,46)$ & $38(33,43)$ \\
\hline $30-39$ & $26(19,35)$ & $31(24,40)$ & $26(22,30)$ \\
\hline $40-49$ & $29(23,37)$ & $14(10,21)$ & $13(10,16)$ \\
$\geq 50$ & $32(25,40)$ & $16(10,24)$ & $24(20,29)$ \\
\hline Female & $39(33,46)$ & $39(32,47)$ & $47(42,51)$ \\
\hline Race/ethnicity & & $10(7,15)$ & $11(8,15)$ \\
\hline Black & $54(45,62)$ & $64(55,72)$ & $78(73,82)$ \\
White & $28(22,36)$ & $26(19,35)$ & $11(8,15)$
\end{tabular}

Primary Payer

\begin{tabular}{lllr} 
Medicaid & $28(22,34)$ & $36(27,45)$ & $34(29,40)$ \\
\hline Medicare & $13(7,23)$ & $5(3,8)$ & $13(10,17)$ \\
\hline Private & $16(11,23)$ & $24(17,34)$ & $19(15,23)$ \\
Uninsured & $24(19,30)$ & $16(10,26)$ & $17(14,20)$ \\
\hline Other & $19(13,27)$ & $12(8,17)$ & $17(12,23)$ \\
\hline Homelessness & $10(6,17)$ & $91(83,96)$ & $88(82,93)$ \\
\hline Urban region & $96(92,98)$ & & $22(18,26)$ \\
\hline US Region & $16(11,23)$ & - & $24(20,29)$ \\
\hline Northeast & $20(14,26)$ & - & $27(23,33)$ \\
\hline Midwest & $49(40,59)$ & $28(22,36)$ & $27(21,32)$ \\
\hline South & $15(10,21)$ & $60(51,68)$ & $24(20,29)$ \\
\hline West & $28(22,36)$ & $26(19,35)$ & $13(10,17)$
\end{tabular}

Source: National Hospital Ambulatory Medical Care Survey. Cell sizes with $<30$ unweighted visits or $>30 \%$ relative standard error not included. Visits were mutually exclusive for drug type, as visits associated with two or more drug-categories were excluded. Multimorbidity as the presence of two or more comorbidities assessed by NHAMCS (including Alzheimer's dementia, alcohol use disorder, asthma, cancer, cerebrovascular disease, chronic obstructive pulmonary disease, coronary artery disease, depression, diabetes, chronic kidney disease, end stage renal disease, venous thromboembolism, HIV/AIDS, hypertension, obesity, obstructive sleep apnea, osteoporosis, and substance use disorders).

Regarding chief presenting concerns, psychiatric concerns were more common in cocaine- and psychostimulant-related visits (cocaine $31 \%$, psychostimulant $50 \%$, opioid $25 \%$; $<$ 0.001). Cardiopulmonary concerns were also more common in cocaine- and psychostimulant-related visits (cocaine $33 \%$, psychostimulant $23 \%$, opioid $12 \%$; $<0.001$ ) concerns than opioid-related visits (Table 2 ). Drug-toxicity/withdrawal concerns were more common in opioid-related visits (cocaine $36 \%$, psychostimulant $32 \%$, opioid $49 \%$; $<<0.001$ ). Cocaine-related visits had a higher proportion of co-occurring alcoholrelated diagnoses in the same ED visit (cocaine 19\%, psychostimulant $6 \%$, opioid $7 \%$; $p<0.001$ ). 
Table 2

Presenting concerns, clinical management, and disposition of national emergency department visits related to cocaine, psychostimulant, or opioid use, $2008-2018$

\section{Weighted \% $(95 \% \mathrm{Cl})$}

$\begin{array}{lll}\text { Cocaine-related visits } & \text { Psychostimulant-related visits } & \text { Opioid-related visits } \\ (n=1,406,000) & (n=1,590,000) & (n=4,125,000)\end{array}$

\section{Chief presenting concern(s)}

\begin{tabular}{llll} 
Psychiatric & $31(24,38)$ & $50(42,58)$ & $25(21,30)$ \\
Neurologic & $7(4,11)$ & $7(4,12)$ & $7(4,10)$ \\
\hline Cardiopulmonary & $33(26,41)$ & $23(17,31)$ & $12(10,16)$ \\
Drug toxicity/withdrawal & $36(30,43)$ & $32(25,40)$ & $49(43,54)$
\end{tabular}

\section{Co-occurring Drug Related Diagnoses}

\begin{tabular}{llll} 
Alcohol-related diagnosis & $19(15,25)$ & $6(4,10)$ & $7(5,9)$ \\
\hline Other drug-related diagnosis & $9(6,13)$ & $9(6,14)$ & $9(7,12)$
\end{tabular}

\section{Diagnostic testing}

\begin{tabular}{llll} 
Blood alcohol concentration & $20(15,27)$ & $20(14,29)$ & $16(12,19)$ \\
Cardiac monitoring & $24(18,32)$ & $13(8,20)$ & $12(9,16)$ \\
\hline Cardiac biomarkers & $23(16,32)$ & $11(7,16)$ & $7(5,9)$ \\
\hline Electrocardiogram & $51(43,59)$ & $34(27,41)$ & $29(24,33)$ \\
\hline Urine toxicology & $56(47,64)$ & $42(34,51)$ & $35(30,41)$ \\
\hline Any imaging & $54(46,61)$ & $35(27,44)$ & $35(30,41)$ \\
Any X-ray & $44(37,52)$ & $28(21,37)$ & $26(20,31)$ \\
Any CT Scan & $17(12,23)$ & $14(8,21)$ & $13(10,17)$
\end{tabular}

Administered medications

$\begin{array}{llll}\text { Atypical antipsychotics } & 6(4,11) & 13(8,20) & 2(1,3) \\ \text { Benzodiazepines } & 19(14,25) & 33(26,42) & 15(12,19) \\ \text { Naloxone } & - & - & 13(10,17) \\ \text { Opioids } & 17(11,24) & 9(4,17) & 14(11,18)\end{array}$

\section{Disposition}

\begin{tabular}{|c|c|c|c|}
\hline Treat and release & $58(51,65)$ & $63(55,70)$ & $68(63,72)$ \\
\hline Left before treatment complete & $3(1,5)$ & $2(1,6)$ & $3(2,6)$ \\
\hline Transferred to psychiatric facility & $6(3,13)$ & $10(6,17)$ & $5(3,7)$ \\
\hline Admitted & $16(12,22)$ & $9(6,13)$ & $16(13,20)$ \\
\hline
\end{tabular}

Source: National Hospital Ambulatory Medical Care Survey. Cell sizes with $<30$ unweighted visits or $>30 \%$ relative standard error not included. Visits were mutually exclusive for drug type, as visits associated with two or more drug-categories were excluded. Chief presenting concerns defined using top three "reason for visit" codes. Visits could contribute to more than one category of chief presenting concerns.

The most common chief presenting concerns varied across groups. The most common chief presenting concern for opioid-related visits were "adverse effect of drug abuse" (27.9\% of opioid-related visits), "drug detoxification" (6.0\%), and "abnormal drug usage" (5.7\%) (Appendix 3). The most common chief presenting concerns for cocaine-related visits were "chest pain" (27.2\% of cocaine-related visits), "other problems relating to psychosis" (7.9\%), and "abdominal pain" (5.7\%). For psychostimulant-related visits, most common chief presenting concerns were "chest pain" (10.4\% of psychostimulant-related visits), "abnormal drug usage" (9.3\%), and "other symptoms related to psychosis" (8.7\%).

Cocaine-related visits had the highest utilization of diagnostic testing, especially cardiovascular testing (e.g., cardiac biomarkers and monitoring). Psychostimulant-related visits had more administration of chemical restraint medications and more transfers to psychiatric facilities (cocaine $6 \%$, psychostimulant $10 \%$, opioid $5 \%$; $<<0.001)$. 
Unadjusted odd ratios (OR) showing associations between drug type and presenting concerns are shown in Table 3. Adjusting for age, sex, race/ethnicity, and homelessness, psychostimulant-related visits had greater odds of presenting with a psychiatric chief concern compared to opioid-related visits (adjusted odds ratio [aOR] 2.69; 95\% Cl 1.83-3.95; Table 3). No differences were seen with neurologic chief concerns. Both cocaine- and psychostimulantrelated visits had greater odds of presenting with cardiopulmonary chief concerns compared to opioid-related visits (cocaine aOR $2.95,95 \% \mathrm{Cl} 1.70-5.13$ : psychostimulant aOR $2.46,95 \% \mathrm{Cl} 1.42-4.27)$. In contrast, psychostimulant-related visits had lower odds of presenting with drug toxicity/withdrawal concerns (aOR 0.47, 95\% Cl 0.30-0.73).

Table 3

Associations between drug type and chief presenting concerns among national emergency department visits related to cocaine, psychostimulant, or opioid use, 2008-2018.

\begin{tabular}{|c|c|c|c|c|c|c|c|c|}
\hline & \multicolumn{2}{|c|}{$\begin{array}{l}\text { Psychiatric chief } \\
\text { concerns }\end{array}$} & \multicolumn{2}{|c|}{$\begin{array}{l}\text { Neurologic chief } \\
\text { concerns }\end{array}$} & \multicolumn{2}{|c|}{$\begin{array}{l}\text { Cardiopulmonary chief } \\
\text { concerns }\end{array}$} & \multicolumn{2}{|c|}{$\begin{array}{l}\text { Drug toxicity/ withdrawal chief } \\
\text { concerns }\end{array}$} \\
\hline & OR & $95 \% \mathrm{Cl}$ & OR & $95 \% \mathrm{Cl}$ & OR & $95 \% \mathrm{Cl}$ & OR & $95 \% \mathrm{Cl}$ \\
\hline \multicolumn{9}{|c|}{ Unadjusted analyses } \\
\hline \multicolumn{9}{|l|}{ Drug } \\
\hline Cocaine & 1.32 & $0.85-2.21$ & 0.99 & $0.51-1.95$ & 3.52 & $2.34-5.31$ & 0.60 & $0.42-0.87$ \\
\hline Psychostimulants & 2.99 & $2.04-4.39$ & 1.03 & $0.49-2.17$ & 2.12 & $1.26-3.58$ & 0.49 & $0.32-0.76$ \\
\hline Opioid & Ref & & Ref & & Ref & & Ref & \\
\hline \multicolumn{9}{|l|}{ Adjusted analyses } \\
\hline \multicolumn{9}{|l|}{ Drug } \\
\hline Cocaine & 1.37 & $0.85-2.21$ & 1.05 & $0.87-2.28$ & 2.95 & $1.70-5.13$ & 0.83 & $0.52-1.35$ \\
\hline Psychostimulants & 2.69 & $1.83-3.95$ & 0.92 & $0.36-2.37$ & 2.46 & $1.42-4.26$ & 0.47 & $0.30-0.73$ \\
\hline Opioid & \multicolumn{2}{|l|}{ Ref } & \multicolumn{2}{|l|}{ Ref } & \multicolumn{2}{|l|}{ Ref } & \multicolumn{2}{|l|}{ Ref } \\
\hline
\end{tabular}

\section{Discussion}

In this study using nationally representative ED data, there were significant increases in opioid-related ED visits and psychostimulant-related ED visits, where the latter increased from 2.2 to 12.9 visits per 10,000 population from 2008 to 2018. This finding is consistent with other studies showing increasing national rates of ED visits, hospitalizations, and deaths from psychostimulant overdose. ${ }^{2,4,5,37}$ The increasing use of the ED and other acute care settings is likely linked to rising methamphetamine availability and use. ${ }^{38}$ Data from the National Forensic Laboratory Information System found methamphetamine drug case submissions increased from 2011 to 2019 , with methamphetamine as the most frequently reported drug. ${ }^{39}$ While psychostimulant-related ED visits were predominantly among Western regions in our study, recent data highlights the emergence of psychostimulantrelated overdose deaths in the Midwest and Northeast, suggesting methamphetamine is already becoming a nationwide concern. ${ }^{8,40}$ Increases in cocainerelated ED visits were not significant, potentially due to the exclusion of visits related to opioid and cocaine co-use. Polysubstance use is common in among individuals using cocaine ${ }^{36}$ and other studies found rates of fatal overdoses and ED visits for overdose involving cocaine and opioid use are rising. ${ }^{5,37}$

We found stimulant-related ED visits were less likely to be identified as drug toxicity/withdrawal concerns, underscoring the differences in presentations between stimulant- and opioid-related visits. While the national surge in ED visits and deaths related to opioid overdose is linked to the rise in fentanyl in the drug supply, ${ }^{1,37,41}$ the main drivers of stimulant-related ED visits and overdoses are unclear. Possibilities include increased potency of fluctuating drug supplies, ${ }^{39}$ contamination or co-use with synthetic opioids like fentanyl, ${ }^{42}$ or the cumulative effects of chronic stimulant use over time. ${ }^{33}$ In addition, the term "overdose" may be problematic for stimulants. The "overdose" terminology commonly referring to an acute respiratory event from an episode of opioid use may be problematic when applied to stimulants, as it lacks specificity in capturing the diverse ways in which stimulant toxicity can present. Because of this variety in stimulant-related presentations, acute complications from stimulant use are more likely to comprise a toxidrome of multiple potential symptoms. ${ }^{16,43}$ Addressing acute stimulant toxicity may rely more on clinical management of various symptoms, rather than the development of a single reversal agent like naloxone for opioid overdose.

The ED has become a critical setting in responding to the opioid overdose crisis and addressing the health needs of people who use drugs, particularly among low-income and uninsured populations. ${ }^{44,45}$ EDs have taken up campaigns to distribute naloxone, ${ }^{46-48}$ initiate buprenorphine prescriptions for opioid use disorder treatment, ${ }^{45}$ and develop referral programs to increase linkages to treatment services. ${ }^{49,50}$ Similarly, the ED can become a point of intervention for addressing acute and chronic complications of stimulant use disorders. ${ }^{51}$ While no current medications are approved by the Food and Drug Administration, several medications show early promise. ${ }^{52-54}$ Behavioral treatments such as contingency management and cognitive behavioral 
therapy are effective in reducing stimulant use, though few are able to access these treatment modalities, especially for publicly insured populations where reimbursement is limited. ${ }^{55-57}$ EDs facing a high burden of stimulant-related visits could implement a navigator to facilitate linkages to existing stimulant use treatment programs, similar to what has been done for other substance use disorders, ${ }^{49,58}$ or offer harm reduction kits to reduce preventable complications of drug use. As methamphetamine-related ED visits are associated with longer length of stays and higher costs, ${ }^{14}$ facilitating linkages to stimulant use treatment from the ED could help reduce acute care costs and would align with national policies focused on expanding access to treatment. $^{59}$

Cocaine-related ED visits were predominately made by individuals who were older, male, and Black. Potential reasons include differences in drug supply, disparities in comorbidities, socioeconomic disadvantage, and other factors related to structural racism that can affect health and healthcare access. ${ }^{60,61}$ Complications from cocaine use are disproportionately higher in Black communities, where rates of cocaine-related deaths are comparable to the rates of opioid-related deaths in white individuals. ${ }^{60}$ Yet cocaine-related harms have largely been understudied in recent years, and this is alarming given overdose death rates in Black individuals are rising faster compared to whites. ${ }^{62,63}$ As the country begins to address the rising epidemic of stimulant-related deaths, interventions addressing stimulant use must address racial equity and pay attention to both cocaine and psychostimulant use to avoid further exacerbating existing racial and economic structural disparities. ${ }^{64}$

Both cocaine- and psychostimulant-related ED visits were associated with cardiopulmonary concerns, even after adjusting for factors associated with increased cardiovascular risk. Both cocaine and psychostimulants are known cardiotoxins, and both acute and chronic use can lead to adverse events such as myocardial ischemia, stroke, and heart failure. ${ }^{17,65,66}$ Patients using stimulants who develop chronic cardiovascular conditions such as heart failure, despite being younger, face more severe in-hospital complications with higher rates of readmission compared to patients with non-stimulant-related heart failure. ${ }^{67,68}$ These findings suggest a need for targeted cardiopulmonary interventions for people who present to the ED with stimulant-related diagnoses.

We found psychostimulant-related ED visits were strongly associated with psychiatric concerns, had greater administration of chemical restraint medications, and had more transfers to psychiatric facilities. Single center ED studies have drawn similar conclusions, ${ }^{14,15}$ and our study using national data adds generalizability to these findings. Use of methamphetamine not only precipitates psychotic symptoms, but methamphetamine use also exacerbates underlying psychiatric illness. ${ }^{14,69}$ Paired with high rates of homelessness seen in patients with stimulant-related ED visits, these findings are especially relevant for urban areas where addressing acute psychiatric presentations and homelessness are pressing concerns.

Several limitations should be considered. We excluded visits that included diagnoses related to both opioid and stimulant use, which may underestimate both the rates of drug-related ED visits and the role of polysubstance use. Prior work has shown combined opioid and stimulant overdose deaths often present similar to opioid-only overdose deaths, ${ }^{33}$ and this may apply also be true for ED visits related to both opioid and stimulant use. The transition from $I C D-9-C M$ to $I C D-10-C M$ codes starting in 2015 potentially affected trends, as increasing rates of drug-related visits during this period could be attributed to this transition. ${ }^{34}$ However, the continued rise across all drug-related ED visits after 2015 provides reassurance that our trends have validity. Diagnostic coding of visits may not have accurately captured all-drug related ED visits, as it often relies on the judgment of the treating physician which may introduce misclassification. Though this preliminary data is needed to set the stage for longitudinal prospective studies to validate these findings. Finally, we could not distinguish between visits related to methamphetamine versus other psychostimulants, as there are no methamphetamine-specific $I C D$ codes. Previous studies have shown that psychostimulant-related $I C D$ codes have high positive predictive values for methamphetamine use, particularly in acute care settings. ${ }^{2,6,7,35}$

\section{Conclusions}

Psychostimulant-related ED visits increased substantially from 2008 to 2018 . Cocaine- and psychostimulant-related ED visits differ in presentation and management from opioid-related ED visits. They are less often identified as related to drug toxicity/withdrawal and require more interventions to address cardiopulmonary and psychiatric complications.

\section{Abbreviations}

ED

emergency department; ICD:international classification of diseases; NCHS:National Center of Health Statistics; NHAMCS:National Hospital Ambulatory Medical Care Survey; RFV:reason for visit

\section{Declarations}

Ethics approval: As the study uses publicly available datasets, the University of California San Francisco Institutional Review Board exempted this study from review.

Consent for Publication: Not applicable.

Availability of data and materials: All data is made publicly available through the National Center of Health Statistics and can be accessed through their website at https://www.cdc.gov/nchs/ahcd/index.htm 
Prior presentations: None.

Potential Competing Interest(s): KTL has received research funding unrelated to this work from the California Department of Health Care Services, Public Health Institute, Sierra Health Foundation, and Substance Abuse and Mental Health Services Administration. POC serves on the Board of Scientific Counselors, National Center for Injury Prevention and Control, Centers for Disease Control and Prevention.

Funding: LWS was supported by the UCSF National Clinical Scholars Program and the San Francisco Veteran Affairs Medical Center. TDM has received training support from the National Institute on Drug Abuse (T32 DA007250). POC was supported by the National Institute on Drug Abuse (K24DA042720). The funding agencies had no role in study design, data collection, analysis, the decision to publish, or the preparation of the manuscript.

Author contributions: LWS and PC conceived the study and designed the study. LWS analyzed the data. TDM provided statistical advice on data analysis. All authors contributed to data interpretation. LWS and PC drafted the article, and all authors contributed substantially to its revision.

Acknowledgements: We thank Christopher Cairns and Loredana Santo at the Division of Health Care Statistics at the National Center for Health Statistics, and Dr. John Neuhaus at the UCSF Epidemiology \& Biostatistics, for their assistance with the data. We would also like to thank the UCSF National Clinical Scholars Program for their support of this work.

\section{References}

1. Holland KM, Jones C, Vivolo-Kantor AM, et al. Trends in US Emergency Department Visits for Mental Health, Overdose, and Violence Outcomes Before and During the COVID-19 Pandemic. JAMA Psychiatry. Published online 2021:8.

2. Winkelman TNA, Admon LK, Jennings L, Shippee ND, Richardson CR, Bart G. Evaluation of Amphetamine-Related Hospitalizations and Associated Clinical Outcomes and Costs in the United States. JAMA Netw Open. 2018;1(6):e183758. doi:10.1001/jamanetworkopen.2018.3758

3. Jones CM, Olsen EO, O'Donnell J, Mustaquim D. Resurgent Methamphetamine Use at Treatment Admission in the United States, 2008-2017. Am J Public Health. 2020;110(4):509-516. doi:10.2105/AJPH.2019.305527

4. Vivolo-Kantor AM, Hoots BE, Seth P, Jones CM. Recent trends and associated factors of amphetamine-type stimulant overdoses in emergency departments. Drug Alcohol Depend. 2020;216:108323. doi:10.1016/j.drugalcdep.2020.108323

5. Hoots B, Vivolo-Kantor A, Seth P. The rise in non-fatal and fatal overdoses involving stimulants with and without opioids in the United States. Addiction. 2020;115(5):946-958. doi:https://doi.org/10.1111/add.14878

6. Crane EH. Highlights of the 2011 Drug Abuse Warning network (DAWN) Findings on Drug-Related Emergency Department Visits. Published online February 22, 2013. doi:10.1037/e515452011-001

7. Substance Abuse and Mental Health Services Administration. Drug Abuse Warning Network, 2011: National Estimates of Drug-Related Emergency Department Visits. Substance Abuse and Mental Health Services Administration; 2013:100.

https://www.samhsa.gov/data/sites/default/files/DAWN2k11ED/DAWN2k11ED/DAWN2k11ED.pdf

8. Mattson CL, Tanz LJ, Quinn K, Kariisa M, Patel P, Davis NL. Trends and Geographic Patterns in Drug and Synthetic Opioid Overdose Deaths - United States, 2013-2019. MMWR Morb Mortal Wkly Rep. 2021;70:202-207. doi:10.15585/mmwr.mm7006a4

9. Hedegaard H, Bastian BA, Trinidad JP, Spencer M, Warner M. Drugs Most Frequently Involved in Drug Overdose Deaths: United States, $2011-2016$. Natl Vital Stat Rep. 2018;67(9):1-14.

10. Centers for Disease Control and Prevention. Health Alert Network (HAN) Health Advisory HANO0438. Centers for Disease Control and Prevention; 2020. Accessed February 8, 2021. https://emergency.cdc.gov/han/2020/han00438.asp

11. Black JC, Bau GE, Iwanicki JL, Dart RC. Association of Medical Stimulants With Mortality in the US From 2010 to 2017. JAMA Internal Medicine. Published online February 1, 2021. doi:10.1001/jamainternmed.2020.7850

12. Riley ED, Cohen J, Shumway M. Overdose Fatality and Surveillance as a Method for Understanding Mortality Trends in Homeless Populations. JAMA Intern Med. 2013;173(13):1264. doi:10.1001/jamainternmed.2013.6838

13. Derlet RW, Albertson TE. Emergency department presentation of cocaine intoxication. Annals of Emergency Medicine. 1989;18(2):182-186. doi:10.1016/S0196-0644(89)80111-8

14. Murphy CE, Wang RC, Coralic Z, Lai AR, Raven M. Association Between Methamphetamine Use and Psychiatric Hospitalization, Chemical Restraint, and Emergency Department Length of Stay. Acad Emerg Med. 2020;27(11):1116-1125. doi:10.1111/acem.14094

15. Jones R, Woods C, Usher K. Rates and features of methamphetamine-related presentations to emergency departments: An integrative literature review. Journal of Clinical Nursing. 2018;27(13-14):2569-2582. doi:10.1111/jocn.14493

16. Kaye S, Darke S. Non-fatal cocaine overdose among injecting and non-injecting cocaine users in Sydney, Australia. Addiction. 2004;99(10):13151322. doi:10.1111/j.1360-0443.2004.00875.x

17. Afonso L, Mohammad T, Thatai D. Crack Whips the Heart: A Review of the Cardiovascular Toxicity of Cocaine. The American Journal of Cardiology. 2007;100(6):1040-1043. doi:10.1016/j.amjcard.2007.04.049

18. Centers for Disease Control and Prevention. NAMCS/NHAMCS-Scope and Sample Design.; 2019. Accessed April 14, 2020.

https://www.cdc.gov/nchs/ahcd/ahcd_scope.htm 
19. Centers for Disease Control and Prevention. NAMCS/NHAMCS-Estimation Procedures.; 2019. Accessed April 14, 2020.

https://www.cdc.gov/nchs/ahcd/ahcd_estimation_procedures.htm

20. National Center for Health Statistics. 2014 NHAMCS Micro-Data File Documentation. National Center for Health Statistics; 2014. Accessed April 14, 2020. https://data.nber.org/nhamcs/docs/nhamcsed2014.pdf

21. Hsiao C-J. Understanding and Using NAMCS and NHAMCS Data: Data Tools and Basic Programming Techniques. Presented at the: National Conference for Health Statistics; August 16, 2010.

22. National Center for Health Statistics. 2015 NHAMCS Micro-Data File Documentation:; 2015.

23. Centers for Disease Control Prevention. ICD - ICD-9-CM - International Classification of Diseases, Ninth Revision, Clinical Modification. Centers for Disease Control and Prevention. Published March 1, 2019. Accessed February 28, 2021. https://www.cdc.gov/nchs/icd/icd9cm.htm

24. Centers for Disease Control Prevention. ICD - ICD-10-CM - International Classification of Diseases, Tenth Revision, Clinical Modification. Centers for Disease Control and Prevention. Published January 26, 2021. Accessed February 28, 2021. https://www.cdc.gov/nchs/icd/icd10cm.htm

25. National Center for Health Statistics. Diagnosis Master Category List, 2016. Centers for Disease Control and Prevention; 2016.

26. Barnett ML, Song Z, Landon BE. Trends in Physician Referrals in the United States, 1999-2009. Arch Intern Med. 2012;172(2):163. doi:10.1001/archinternmed.2011.722

27. Navickas R, Petric V-K, Feigl AB, Seychell M. Multimorbidity: What do we know? What should we do? J Comorb. 2016;6(1):4-11. doi:10.15256/joc.2016.6.72

28. Boyd RW, Lindo EG, Weeks LD, McLemore MR. On Racism: A New Standard For Publishing On Racial Health Inequities. Health Affairs Blog. Published online July 2, 2020. doi:10.1377/hblog20200630.939347

29. Burt C, Arispe I. Characteristics of Emergency Departments Serving High Volumes of Safety-Net Patients: United States, 2000. National Center for Health Statistics; 2004.

30. Koch H, Campbell WH. The collection and processing of drug information: National Ambulatory Medical Care Survey. United States, 1980. Vital Health Stat 2. 1982;(90):1-90.

31. McCaig LF, Burt CW. Understanding and Interpreting the National Hospital Ambulatory Medical Care Survey: Key Questions and Answers. Annals of Emergency Medicine. 2012;60(6):716-721.e1. doi:10.1016/j.annemergmed.2012.07.010

32. StataCorp. Stata Survey Data Reference Manual: Release 15. Published online 2017. Accessed April 15, 2021. https://www.stata.com/manuals/svysvy.pdf

33. Turner C, Chandrakumar D, Rowe C, Santos G-M, Riley ED, Coffin PO. Cross-sectional cause of death comparisons for stimulant and opioid mortality in San Francisco, 2005-2015. Drug and Alcohol Dependence. 2018;185:305-312. doi:10.1016/j.drugalcdep.2017.12.030

34. Krive J, Patel M, Gehm L, et al. The Complexity and Challenges of the ICD-9-CM to ICD-10-CM Transition in Emergency Departments. Am J Emerg Med. 2015;33(5):713-718. doi:10.1016/j.ajem.2015.03.001

35. Shearer RD, Shippee ND, Winkelman TNA. Characterizing trends in methamphetamine-related health care use when there is no ICD code for "methamphetamine use disorder." Journal of Substance Abuse Treatment. 2021;127:108369. doi:10.1016/j.jsat.2021.108369

36. Liu Y, Williamson V, Setlow B, Cottler LB, Knackstedt LA. The importance of considering polysubstance use: lessons from cocaine research. Drug and Alcohol Dependence. 2018;192:16-28. doi:10.1016/j.drugalcdep.2018.07.025

37. Hedegaard H, Miniño AM, Warner M. Drug Overdose Deaths in the United States, 1999-2019. National Center for Health Statistics; $2020: 8$.

38. Lipari RN. Key Substance Use and Mental Health Indicators in the United States: Results from the 2018 National Survey on Drug Use and Health. Published online 2018:82.

39. Drug Enforcement Administration. National Forensic Laboratory Information System: NFLIS-Drug Mid Year Report 2019. US Department of Justice, Drug Enforcement Administration; 2020.

40. Howell BA, Bart G, Wang EA, Winkelman TNA. Service Involvement Across Multiple Sectors Among People Who Use Opioids, Methamphetamine, or Both, United States-2015-2018. Med Care. 2021;59(3):238-244. doi:10.1097/MLR.0000000000001460

41. Baldwin GT, Seth P, Noonan RK. Continued Increases in Overdose Deaths Related to Synthetic Opioids: Implications for Clinical Practice. JAMA. Published online February 11, 2021. doi:10.1001/jama.2021.1169

42. Stephenson J. Drug Overdose Deaths Head Toward Record Number in 2020, CDC Warns. JAMA Health Forum. 2020;1(10):e201318. doi:10.1001/jamahealthforum.2020.1318

43. Vasan S, Olango GJ. Amphetamine Toxicity. StatPearls Publishing; 2020. Accessed May 7, 2021. https://www.ncbi.nlm.nih.gov/books/NBK470276/

44. Hawk K, D’Onofrio G. Emergency department screening and interventions for substance use disorders. Addiction Science \& Clinical Practice. 2018;13(1):18. doi:10.1186/s13722-018-0117-1

45. D'Onofrio G, O'Connor PG, Pantalon MV, et al. Emergency Department-Initiated Buprenorphine/Naloxone Treatment for Opioid Dependence. JAMA. 2015;313(16):1636-1644. doi:10.1001/jama.2015.3474

46. Bukata WR, Bedard LA, Hoffman JR, Ranney ML, Whiteside LK, Hawk K. Naloxone prescriptions by emergency physicians. ACEP Resolution 39(14), 2014. Published online 2014. Accessed March 11, 2021. https://www.acep.org/what-we-believe/actions-on-council-resolutions/councilresolution/? rid=5AB946E9-9772-E911-A9AD-9BD2C184F805

Page $10 / 12$ 
47. Eswaran V, Allen KC, Bottari DC, et al. Take-Home Naloxone Program Implementation: Lessons Learned From Seven Chicago-Area Hospitals. Ann Emerg Med. 2020;76(3):318-327. doi:10.1016/j.annemergmed.2020.02.013

48. Gunn AH, Smothers ZPW, Schramm-Sapyta N, Freiermuth CE, MacEachern M, Muzyk AJ. The Emergency Department as an Opportunity for Naloxone Distribution. West J Emerg Med. 2018;19(6):1036-1042. doi:10.5811/westjem.2018.8.38829

49. D'Onofrio G, Chawarski MC, O'Connor PG, et al. Emergency Department-Initiated Buprenorphine for Opioid Dependence with Continuation in Primary Care: Outcomes During and After Intervention. J Gen Intern Med. 2017;32(6):660-666. doi:10.1007/s11606-017-3993-2

50. Rapp RC, Otto AL, Lane DT, Redko C, McGatha S, Carlson RG. Improving linkage with substance abuse treatment using brief case management and motivational interviewing. Drug Alcohol Depend. 2008;94(1-3):172-182. doi:10.1016/j.drugalcdep.2007.11.012

51. Selby S, Wang D, Murray E, Lang E. Emergency Departments as the Health Safety Nets of Society: A Descriptive and Multicenter Analysis of Social Worker Support in the Emergency Room. Cureus. 2018;10(9):e3247. doi:10.7759/cureus.3247

52. Coffin PO, Santos G-M, Hern J, et al. Effects of Mirtazapine for Methamphetamine Use Disorder Among Cisgender Men and Transgender Women Who Have Sex With Men: A Placebo-Controlled Randomized Clinical Trial. JAMA Psychiatry. Published online December 11, 2019.

doi:10.1001/jamapsychiatry.2019.3655

53. Trivedi MH, Walker R, Ling W, et al. Bupropion and Naltrexone in Methamphetamine Use Disorder. New England Journal of Medicine. 2021;384(2):140-153. doi:10.1056/NEJMoa2020214

54. National Institute of Drug Abuse. What treatments are under development for methamphetamine use and addiction? National Institute on Drug Abuse. Published April 9, 2020. Accessed March 11, 2021. https://www.drugabuse.gov/publications/research-reports/methamphetamine/what-treatmentsare-under-development-methamphetamine-use-addiction

55. Davis DR, Kurti AN, Skelly JM, Redner R, White TJ, Higgins ST. A review of the literature on contingency management in the treatment of substance use disorders, 2009-2014. Preventive Medicine. 2016;92:36-46. doi:10.1016/j.ypmed.2016.08.008

56. Petry NM. Contingency management: what it is and why psychiatrists should want to use it. Psychiatrist. 2011;35(5):161-163. doi:10.1192/pb.bp.110.031831

57. Rawson RA, McCann MJ, Flammino F, et al. A comparison of contingency management and cognitive-behavioral approaches for stimulant-dependent individuals. Addiction. 2006;101(2):267-274. doi:10.1111/j.1360-0443.2006.01312.x

58. Herring A. California Bridge Program Patient Outcomes EvaluationStudy Protocol (Main Study). Public Health Institution; 2019. Accessed March 12, 2021.

https://static1.squarespace.com/static/5c412ab755b02cec3b4ed998/t/5df2839b04b7db043c691f5d/1576174494551/CA+Bridge+Study+Protocol++Main+Study.pdf

59. Office of National Drug Control Policy. The Biden-Harris Administration's Statement of Drug Policy Priorities for Year One. Published online April 1, 2021.

60. Shiels MS, Freedman ND, Thomas D, Berrington de Gonzalez A. Trends in U.S. Drug Overdose Deaths in Non-Hispanic Black, Hispanic, and NonHispanic White Persons, 2000-2015. Ann Intern Med. 2018;168(6):453. doi:10.7326/M17-1812

61. Cano M, Oh S, Salas-Wright CP, Vaughn MG. Cocaine use and overdose mortality in the United States: Evidence from two national data sources, 20022018. Drug Alcohol Depend. 2020;214:108148. doi:10.1016/j.drugalcdep.2020.108148

62. Drake J, Charles C, Bourgeois JW, Daniel ES, Kwende M. Exploring the impact of the opioid epidemic in Black and Hispanic communities in the United States. Drug Science, Policy and Law. 2020;6:2050324520940428. doi:10.1177/2050324520940428

63. Khatri UG, Pizzicato LN, Viner K, et al. Racial/Ethnic Disparities in Unintentional Fatal and Nonfatal Emergency Medical Services-Attended Opioid Overdoses During the COVID-19 Pandemic in Philadelphia. JAMA Netw Open. 2021;4(1):e2034878. doi:10.1001/jamanetworkopen.2020.34878

64. Nunes EV, Levin FR, Reilly MP, El-Bassel N. Medication treatment for opioid use disorder in the age of COVID-19: Can new regulations modify the opioid cascade? Journal of Substance Abuse Treatment. Published online November 2020:108196. doi:10.1016/j.jsat.2020.108196

65. Kevil Christopher G., Goeders Nicholas E., Woolard Matthew D., et al. Methamphetamine Use and Cardiovascular Disease. Arteriosclerosis, Thrombosis, and Vascular Biology. 2019;39(9):1739-1746. doi:10.1161/ATVBAHA.119.312461

66. Baumann BM, Perrone J, Hornig SE, Shofer FS, Hollander JE, Hollander DJ. Cardiac and Hemodynamic Assessment of Patients with CocaineAssociated Chest Pain Syndromes. Journal of Toxicology: Clinical Toxicology. 2000;38(3):283-290. doi:10.1081/CLT-100100933

67. Shetty S, Malik AH, Ali A, Yang YC, Briasoulis A, Alvarez P. Characteristics, trends, outcomes, and costs of stimulant-related acute heart failure hospitalizations in the United States. International Journal of Cardiology. Published online January 31, 2021. doi:10.1016/j.ijcard.2021.01.060

68. Nishimura M, Ma J, Fox S, et al. Characteristics and Outcomes of Methamphetamine Abuse Among Veterans With Heart Failure. American Journal of Cardiology. 2019;124(6):907-911. doi:10.1016/j.amjcard.2019.05.068

69. Schultz BR, Lu BY, Onoye JM, Toohey TP. High Resource Utilization of Psychiatric Emergency Services by Methamphetamine Users. Hawaii J Med Public Health. 2018;77(12):312-314.

70. Thadani T. Mayor Breed wants to turn an empty San Francisco office into a drug sobering center. San Francisco Chronicle. Published April $13,2021$. Accessed April 15, 2021. https://www.sfchronicle.com/local-politics/article/Mayor-Breed-wants-to-turn-an-empty-San-Francisco-16096092.php

\section{Figures}

Page $11 / 12$ 


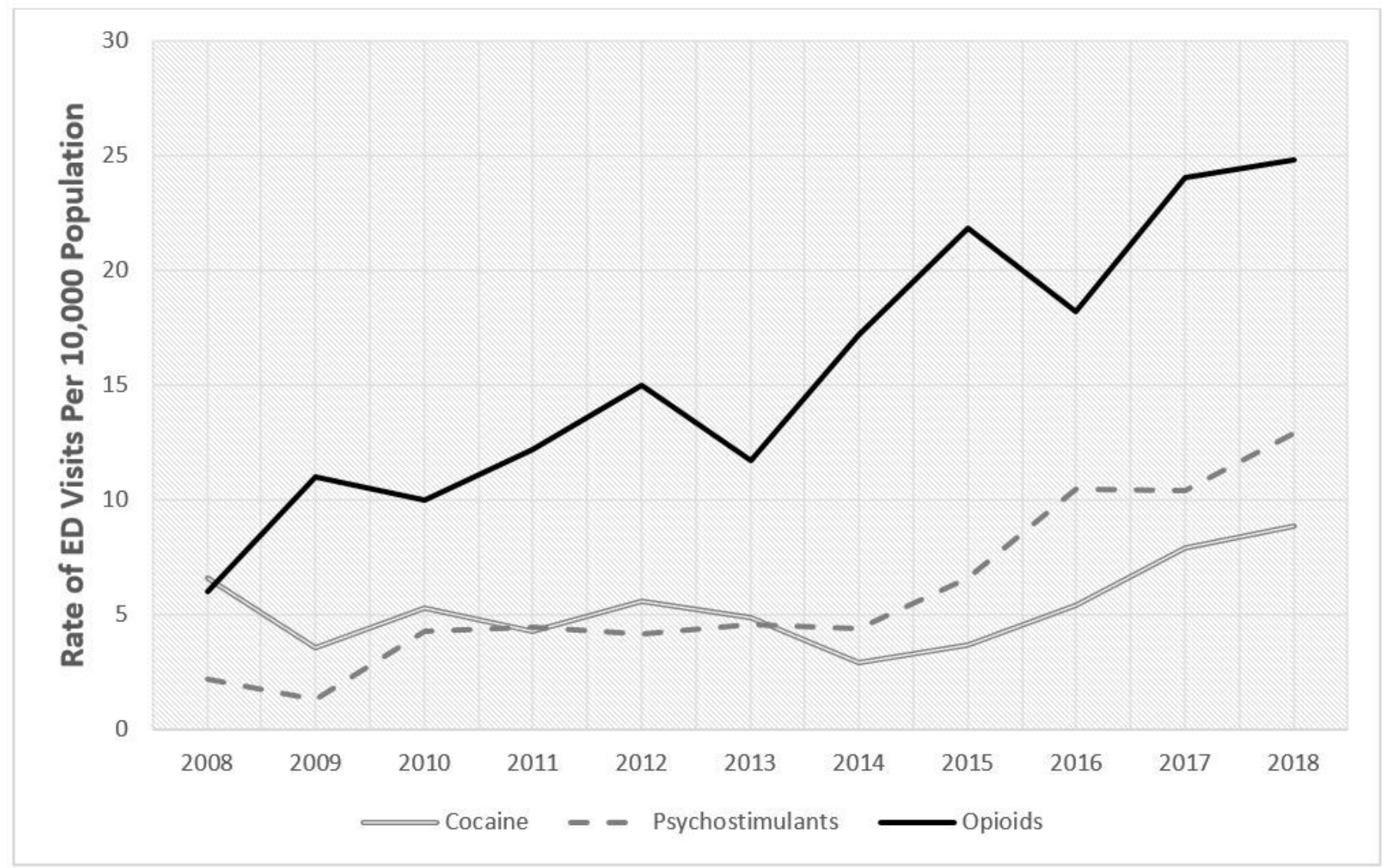

\section{Figure 1}

Annual trends in rates of national emergency department visits related to cocaine, psychostimulant, or opioid use, 2008-2018. Emergency department visits categorized by drug-type if any of the top three ICD9-CM/ICD10-CM diagnoses codes were related to opioid, cocaine, or psychostimulant use. Visits were mutually exclusive for drug type, as visits associated with two or more drug-categories were excluded. Rates were calculated by dividing weighted number of visits in each year by US Census Bureau estimates of civilian, noninstitutionalized adults aged 18 and older for that year. All rates per 10,000 population. Source: National Hospital Ambulatory Medical Care Survey.

\section{Supplementary Files}

This is a list of supplementary files associated with this preprint. Click to download.

- Appendix.docx 